\title{
Trapping mechanisms of persistent photocurrent in GaN-based MSM photodetectors
}

\author{
B. Poti ${ }^{* a}$, A. Passaseo ${ }^{a}$, M. Lomascolo ${ }^{b}$, R. Cingolani ${ }^{a}$ and M. De Vittorio ${ }^{a}$

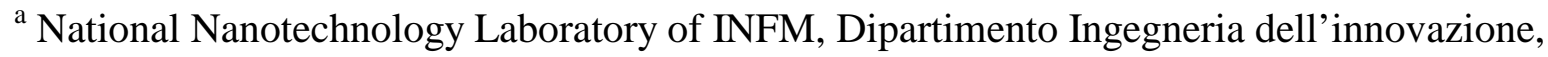 \\ Università di Lecce,I-73100, Italy \\ ${ }^{\mathrm{b}}$ IMM-CNR, Istituto per la Microelettronica ed i Microsistemi, Sez. di Lecce, Via per Arnesano, \\ I-73100 Lecce, Italy
}

\begin{abstract}
The trapping mechanisms at the origin of the persistent photocurrent effects in GaN-based devices have been studied on different time scales by characterizing a low barrier metal-semiconductor-metal GaN-based photodetector in the temperature range between room temperature and $500 \mathrm{~K}$. The active material of the metal-semiconductor-metal device consists of a thin film of GaN grown by metal organic chemical vapour deposition. The Arrhenius plots obtained by the analysis of the decay times of the photocurrent as a function of the temperature on time scales from millisecond up to hours allowed us to calculate the activation energies of the mechanisms responsible for the persistent photocurrent. The activation energies derived from the decay times on the time scale of hours have been attributed to gallium vacancies $\left(\mathrm{V}_{\mathrm{Ga}}\right)$, gallium antisites $\left(\mathrm{Ga}_{\mathrm{N}}\right)$ and carbon impurities, whereas $\mathrm{GaN}$ excitonic resonances resulted to be responsible for the persistent photocurrent on the millisecond time scale. Finally, the influence of the decay times has been correlated with the photocurrent gain of the device, which resulted to be as high as $4.1 \times 10^{5}$ at RT and $0.85 \times 10^{5}$ at $450 \mathrm{~K}$.
\end{abstract}

Keywords: UV detectors, GaN, high temperature, persistent photocurrent, MOCVD, trapping mechanisms, photocurrent gain, gallium vacancies, gallium antisites, carbon impurities.

\section{INTRODUCTION}

Gallium Nitride and its ternary alloys have attracted a large interest in the last years by virtue of their excellent mechanical, electrical and optical properties that offer great potential for applications in high-power/high-temperature electronic and optoelectronic devices ${ }^{1,2}$. However, the presence of phenomena strictly related to the material quality of the device, like yellow luminescence ${ }^{3,5}$, optical quenching ${ }^{6,7}$ and persistent photocurrent $(\mathrm{PPC})^{8-10}$, independent from the growth techniques, suggest that improvements are still necessary to produce layers with low density of defects. Significant concentrations of shallow and deep levels are found in samples epitaxially grown both by metal-organic chemical vapor deposition (MOCVD) and by molecular beam epitaxy (MBE). These native and impurity defects, acting as traps, represent one of the main mechanisms responsible for the PPC, which severely limits the bandwidth of GaNbased UV photodetectors.

In the literature, the origin of the PPC phenomenon is still contradictory. Different trapping mechanisms have been considered at the origin of the PPC, such as $(\mathrm{AX})^{11}$, or $(\mathrm{DX})^{12,13}$ centers, defects with bistable character ${ }^{14}$, random potential fluctuations due to nonstoichiometry ${ }^{15}$, defects at substrate heterointerface $(\mathrm{GaN} / \mathrm{sapphire})^{10}$, and the unintentionally incorporated cubic crystallites in hexagonal GaN $(\mathrm{h}-\mathrm{GaN})^{16}$. Different intrinsic and extrinsic defects have been proposed to be responsible for the PPC. Nitrogen antisites $\left(\mathrm{N}_{\mathrm{Ga}}\right)^{17}$, gallium antisites $\left(\mathrm{Ga}_{\mathrm{N}}\right)^{18}$, gallium vacancies $\left(\mathrm{V}_{\mathrm{Ga}}\right)^{19}$, nitrogen vacancies $\left(\mathrm{V}_{\mathrm{N}}\right)^{20}$ and carbon impurity ${ }^{21}$ are the favoured candidate.

*beatrice.poti@unile.it; phone +39.0832.297213; fax +39.0832.326351 
In this work, in order to clarify the origin of the PPC, we have characterized a low barrier metal-semiconductor-metal (MSM) GaN-based photodetector in the temperature range between room temperature (RT) and $500 \mathrm{~K}$. The active material of the MSM device consists of a thin film of GaN grown by MOCVD. Some of the trapping mechanisms which are at the origin of the PPC effects in GaN-based devices have been studied on different time scales. The Arrhenius plots obtained by the analysis of the decay times of the photocurrent as a function of the temperature on time scales from millisecond up to hours allowed us to calculate the activation energies of the mechanisms responsible for the PPC. Finally, the influence of the decay times has been correlated to the photocurrent gain of the device.

\section{METHODOLOGY}

The samples investigated in this study were grown in a horizontal low pressure-metalorganic chemical vapor deposition system (Aixtron $200 \mathrm{AlX}$ rf), equipped with a rotating substrate holder, with TMGa and pure $\mathrm{NH}_{3}$ as source materials; palladium purified $\mathrm{H}_{2}$ was used as a carrier gas. The growth was performed on (0001) c-plane $\mathrm{Al}_{2} \mathrm{O}_{3}$ substrate cleaned in solvents, and then annealed in situ at $1100^{\circ} \mathrm{C}$. After the deposition of a low temperature nucleation layer, a GaN buffer layer $2 \mu \mathrm{m}$ thick was grown at $1150^{\circ} \mathrm{C}$. The background doping density was $5 \times 10^{17} \mathrm{~cm}^{-3}$ (n type). Before realizing the contacts, a MESA has been realised by reactive ion etching by using a $\mathrm{CH}_{4} / \mathrm{H}_{2} / \mathrm{Ar}^{+}$mixture. This procedure was performed in order to reduce leakage currents among adjacent devices. The device consists of two Ti/Au Schottky contacts on a planar surface deposited by electron-beam evaporation. After optical lithography with a negative tone resist, the interdigitated geometry of the contacts has been realized by lift-off procedure (fig. 1). The fingers were $40 \mu \mathrm{m}$ long and $2 \mu \mathrm{m}$ wide with a pitch of $8 \mu \mathrm{m}$. The devices have an optical area of $40 \times 40 \mu \mathrm{m}^{2}$.

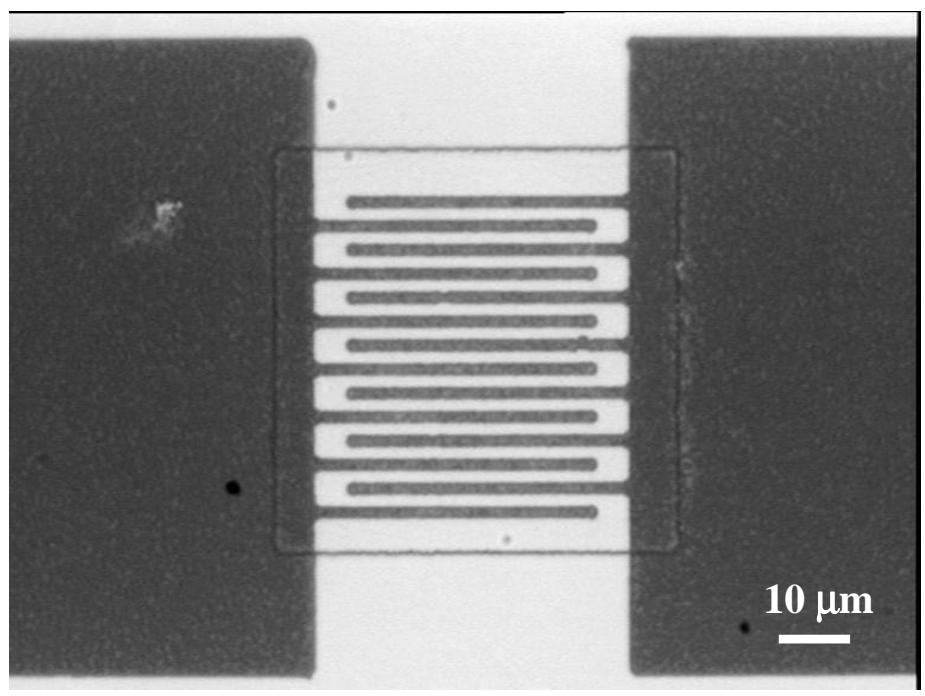

Figure 1 - Top view of the interdigitated geometry of the MSM GaN-based photodetectors.

A focused He-Cd laser $(\lambda=325 \mathrm{~nm})$ was used in order to study the time-resolved photoresponse of the AC photocurrent and to measure the photocurrent gain of the device as a function of the optical power and the temperature. All the measurements were performed at high temperature, in particular in the range between about $300 \mathrm{~K}$ and $500 \mathrm{~K}$, by positioning the samples on a calibrated hot plate and measuring the temperature with a precision resistance temperature detector (RTD). 


\section{RESULTS AND DISCUSSION}

In order to clarify the mechanisms responsible for the PPC on long time scale, the decay kinetic of the photocurrent in the temperature range $300 \mathrm{~K}-500 \mathrm{~K}$ has been studied. In figure 2 we display a representative photocurrent decay curve to a light pulse on long time scale (hours) recorded at $373 \mathrm{~K}$ under bias of $10 \mathrm{~V}$. As can be observed, after the switching-off of the laser pulse, a strong PPC is present in the device later than thousands of seconds even at high temperatures. The experimental decay curves have been fitted (solid line in figure 2) with a stretched-exponential function ${ }^{22,23}$ with two time constants

$$
I(t)=I_{0}+A e^{-\left(\frac{t}{\tau_{1}}\right)^{\alpha}}+B e^{-\left(\frac{t}{\tau_{2}}\right)^{\beta}}
$$

where $\mathrm{I}_{0}$ is defined as the photocurrent value at the moment of light excitation being removed, $\tau_{1}$ and $\tau_{2}$ are the PPC decay time constants, $\alpha$ and $\beta$ are the decay exponents, and A and B are arbitrary constants.

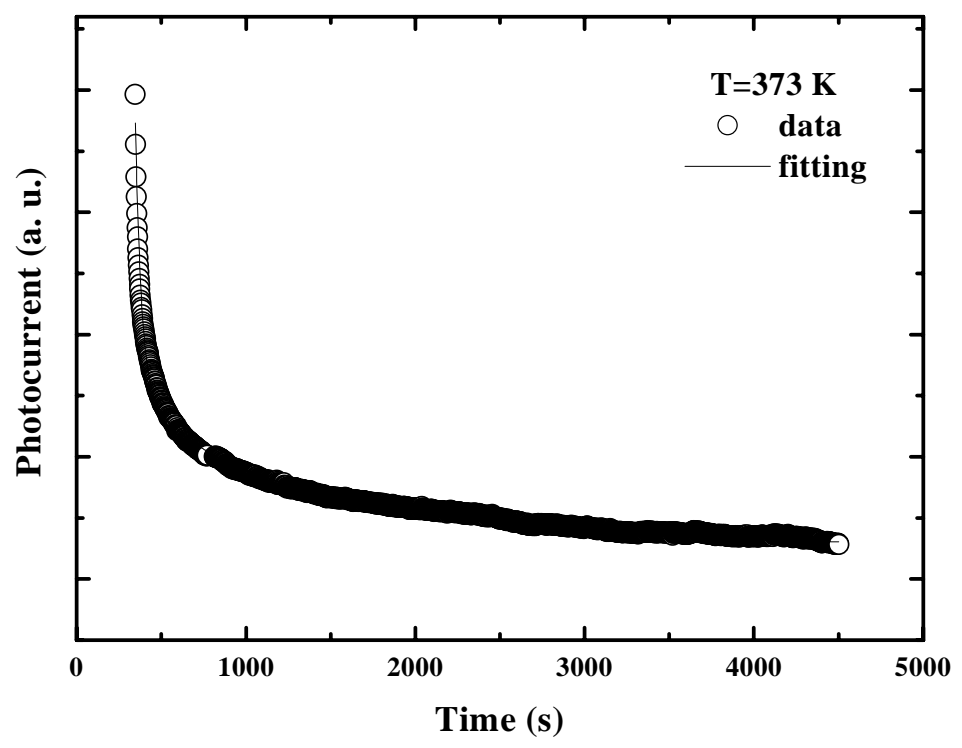

Figure 2-Decay kinetic on long time scale of the MSM GaN-based photodetector at $373 \mathrm{~K}$ under bias of $10 \mathrm{~V}$. The open circles are the experimental data and the solid line is the fitting curve.

As we have already found in the millisecond time scale ${ }^{24}$, the two time constants $\left(\tau_{1}, \tau_{2}\right)$ suggest that at least two main trapping mechanisms, having different release times, are responsible for the PPC on long time scale. By increasing the temperature from $320 \mathrm{~K}$ up to $500 \mathrm{~K}$, the first time constant $\tau_{1}$ decreases of a factor of four from $100 \mathrm{~s}$ down to $25 \mathrm{~s}$, whereas the second time constant $\tau_{2}$ undergoes a reduction as high as five orders of magnitude (from $0.9 \mathrm{~ms}$ down to 33 ns). Figure 3 shows the Arrhenius plots of the two decay times. The linear fits (dotted lines in figures $3 a$ and $3 b$ ) of the two curves allowed us to calculate the activation energies of the two trapping mechanisms related to $\tau_{1}$, $\tau_{2}$ which resulted to be $150 \pm 13 \mathrm{meV}$ and $988 \pm 32 \mathrm{meV}$, respectively. In agreement with the energy levels reported in the literature, we have attributed these values to native defects and impurity levels in GaN. Particularly, the activation energy related to $\tau_{1}$ has been attributed to gallium vacancies $\left(\mathrm{V}_{\mathrm{Ga}}\right)$, which typically is reported to have an activation energy of about 140 
$\mathrm{meV}^{25,26}$. The second activation energy related to $\tau_{2}$ can be attributed to both gallium antisites $\left(\mathrm{Ga}_{\mathrm{N}}\right)$ and carbon impurities, whose typical activation energy is around $1 \mathrm{eV}^{25,27}$, as reported in the literature. The two energy levels in the $\mathrm{GaN}$ energy gap, contributing to the trapping of the photogenerated carriers, give rise to the PPC effects on a time scale of hours.
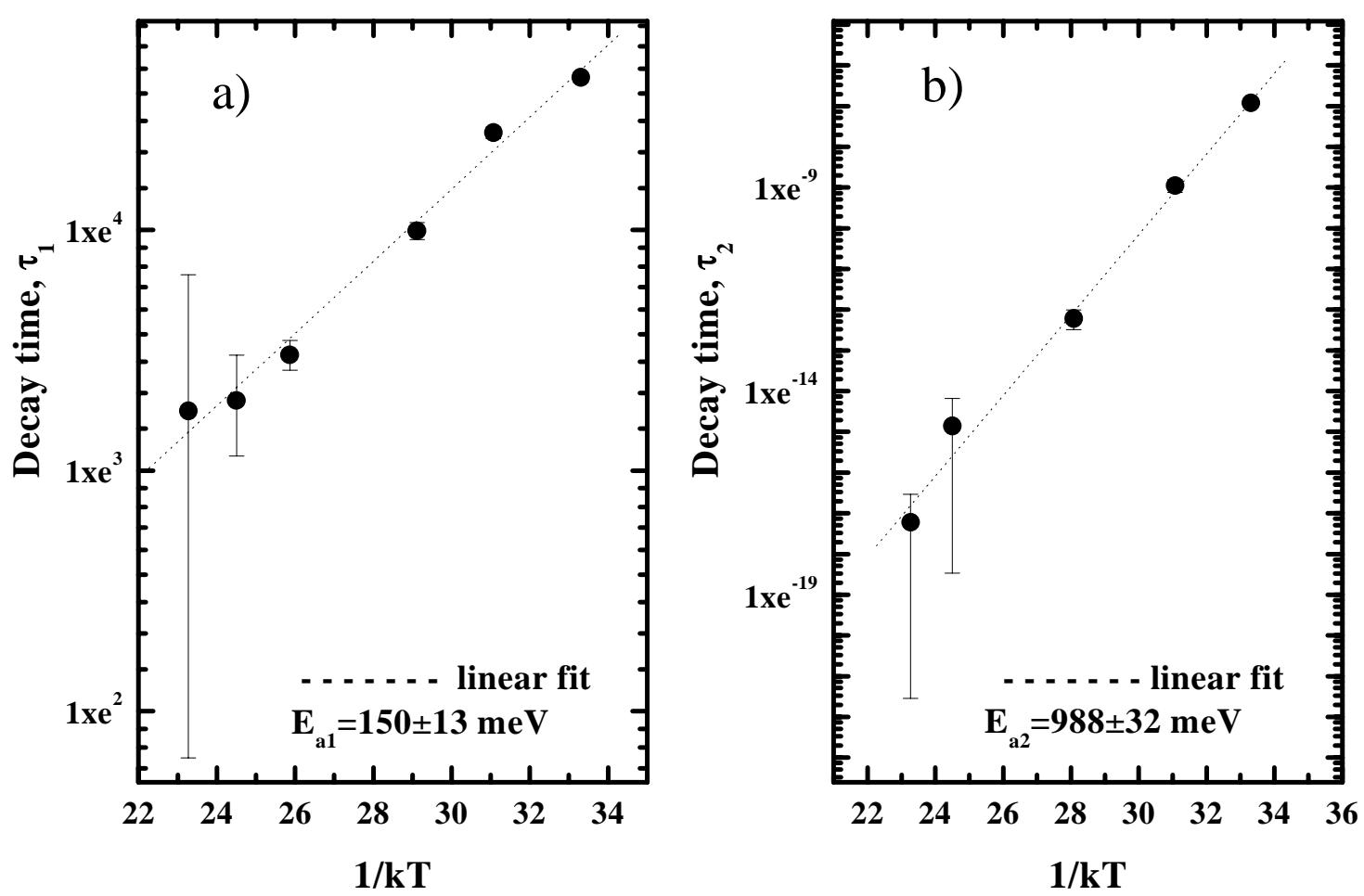

Figure 3-The Arrhenius plots of the PPC decay time constants $\tau_{1}$ (a) and $\tau_{2}$ (b) obtained on long time scale. The corresponding activation energies are about $140 \mathrm{meV}\left(\tau_{1}\right)$ and of about $1 \mathrm{eV}\left(\tau_{2}\right)$, respectively.

The drastic decrease with the temperature of the decay time $\tau_{2}$ suggests that the concentration of high activation energy defects $\left(\mathrm{Ga}_{\mathrm{N}}\right.$ and carbon impurities) is lower than the concentration of low-activation energy defects $\left(\mathrm{V}_{\mathrm{Ga}}\right)$. This is in agreement with the theoretical calculations which predict that in $\mathrm{GaN}$ the formation of $\mathrm{Ga}_{\mathrm{N}}$ and carbon impurities is energetically less favourable than the formation of $\mathrm{V}_{\mathrm{Ga}}{ }^{28}$.

The analysis performed on the millisecond time scale (not reported here) evidences two more activation energies of about $28 \mathrm{meV}$ and $37 \mathrm{meV}^{24}$, which we attributed to two different GaN excitonic transition (type A and probably type C). This shows that in our devices the excitonic resonances provide one of the most important contribution to the PPC on the millisecond time scale at low temperature. Therefore, at temperatures lower than $400 \mathrm{~K}$, PPC effects cannot be avoided even in ideally defect-free $\mathrm{GaN}$ materials. The two constants are reduced by a factor of two with increasing temperature, resulting in the enhancement of the band frequency and in the reduction of the hysteresis error. Nevertheless, independently from the time scale, the increase of the temperature helps to ionize the excitons and the trapped carriers thus reducing the PPC and improving the time response of the device. 
These results show that the PPC effects come from several simultaneous contributions, each one having its own characteristic time. Particularly, the origin of the PPC effects on millisecond time scale is primarily due to the excitonic resonances whereas on long time scale (hours) the origin of this phenomenon derived from the presence in the material of native defects and impurities like gallium vacancies $\left(\mathrm{V}_{\mathrm{Ga}}\right)$, gallium antisites $\left(\mathrm{Ga}_{\mathrm{N}}\right)$ and carbon impurities.

These defects reduces the performances of GaN-based devices in terms of bandwidth. On the other hand the long lifetime of these defects has a positive influence on the gain of the UV detector. In fact, the decay times of the GaN defects affect the photocurrent gain of the device (number of electrons circulating through the sample for absorbed photons per unit time) as shown in the following equation:

$$
g=\frac{\tau_{\text {decay }}}{t_{t r}}=\frac{\tau_{\text {decay }}}{\frac{L}{\mu_{e} E}}
$$

where, $\tau_{\text {decay }}$ represent the decay time of the photocurrent, $t_{t r}$ is the electron transit time and $\mathrm{L}$ is the finger distance. This expression of the photocurrent gain derived from a simple photoconductivity model ${ }^{29}$, holding for low metalsemiconductor $(\mathrm{Ti} / \mathrm{GaN})$ Schottky barriers.

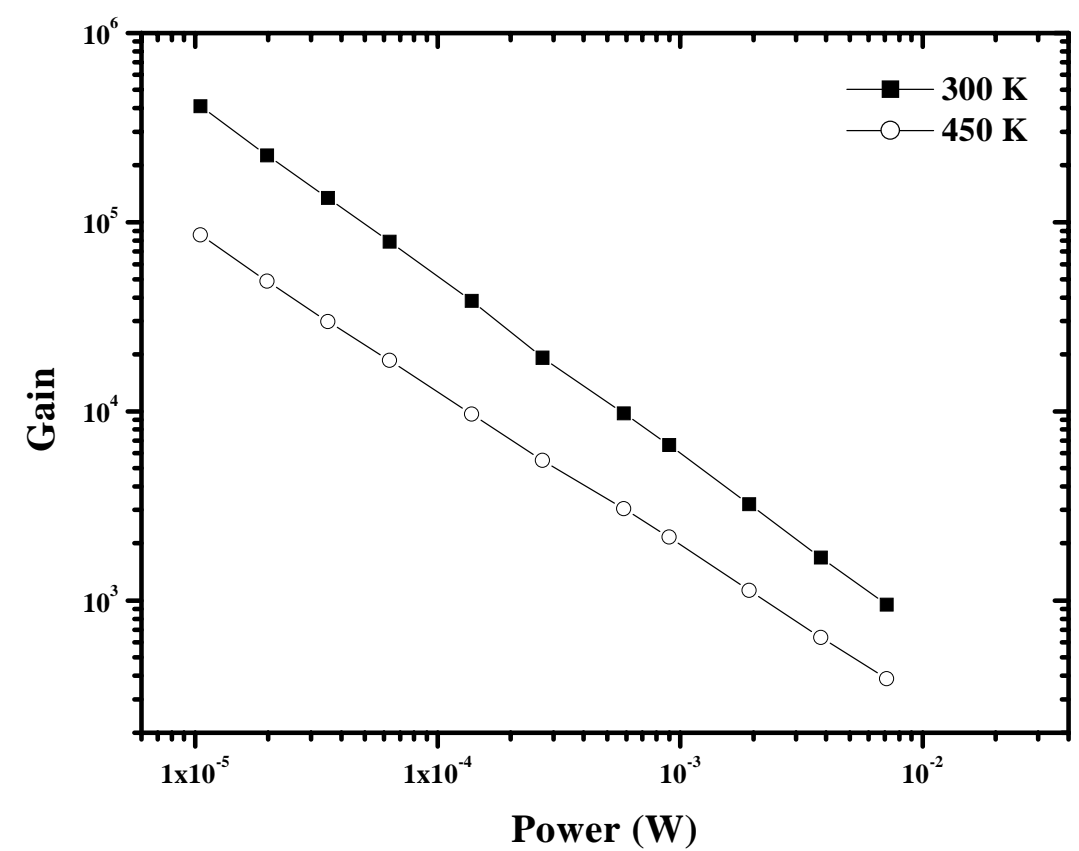

Figure.4 Photocurrent gain versus optical power at different device temperatures for a GaN-based photodetector at $10 \mathrm{~V}$ bias, $325 \mathrm{~nm}$.

In order to estimate the photocurrent gain of the device we have measured the responsivity of the device which is related to the photocurrent gain of the device through the following equation

$$
g=\frac{P_{o p}}{q L G_{L}} R
$$


where $P_{o p}$ is the optical power density and $G_{L}$ the generation rate of the electron-hole pair. The $G_{L}$ was estimated by taking into account the optical reflection of the laser at the air-GaN interface and by assuming that each transmitted photon generates an electron-hole pair.

In figure 4 we show the photocurrent gain as a function of the incident optical power at RT (open circles) and at $450 \mathrm{~K}$ (full squares), at the wavelength of $325 \mathrm{~nm}$. At low optical power $\left(\sim 10^{-5} \mathrm{~W}\right)$ a photocurrent gain as high as $4.1 \times 10^{5}$ has been measured at RT, which decreases down to $0.85 \times 10^{5}$ at $450 \mathrm{~K}$. To our knowledge these are the highest values ever reported in the literature. By increasing the power the photocurrent gain at both RT (open circles) and $450 \mathrm{~K}$ (full squares) is reduced following a $\mathrm{P}^{-\mathrm{k}}$ law, with $\mathrm{k} \sim 0.9$ at $\mathrm{RT}$ and $\mathrm{k} \sim 0.8$ at $450 \mathrm{~K}^{30}$.

The high values of the gain can be directly related to the long decay time of defects such as $\mathrm{V}_{\mathrm{Ga}}, \mathrm{Ga}_{\mathrm{N}}$ and carbon impurities. The gain values measured in our devices can be attributed both to the high stationary photoconductivity lifetime (for low optical power density) and to the short distance between the interdigitated electrodes.

The increase of the temperature leads to a reduction of the photocurrent gain, but it allows the ionization of low activation energy trapping centers, thus improving the bandwidth of the device.

\section{CONCLUSIONS}

In this work we have shown that the origin of the PPC in GaN-based devices comes from several simultaneous contributions, each one having its own characteristic time. The analysis of the decay kinetic of the photocurrent versus temperature reveals that the origin of the PPC effects on millisecond time scale is primarily due to the excitonic resonances whereas on long time scale (hours) the origin of this phenomenon derived from the presence in the optical material of gallium vacancies $\left(\mathrm{V}_{\mathrm{Ga}}\right)$, gallium antisites $\left(\mathrm{Ga}_{\mathrm{N}}\right)$ and carbon impurities. These latter trapping mechanisms are native defects and impurity levels located at about $140 \mathrm{meV}$ and $940 \mathrm{meV}$ above the valence band. Nevertheless, the increase of the temperature allows the ionization of low-energy trapping centers, thus improving the bandwidth of the device. Finally, the influence of the decay times has been correlated to the high photocurrent gain of the device, which resulted to be as high as $4.1 \times 10^{5}$ at RT and $0.85 \times 10^{5}$ at $450 \mathrm{~K}$.

\section{ACKNOWLEDGEMENTS}

We gratefully acknowledge I. Tarantini and G. Epifani for the technical help. 


\section{REFERENCES}

1. M. Razeghi and A. Rogalski, "Semiconductor ultraviolet detectors", J. Appl. Phys. 79 (10), 7433, 1996.

2. D. Walker, E. Monroy, P. Kung, J. Wu, M. Hamilton, F. J. Sanchez, J. Diaz "High-speed, low-noise metalsemiconductor-metal ultraviolet photodetectors based on GaN", Appl. Phys. Lett. 74 (5), 762, 1999.

3. S. J. Chung, O. H. Cha, Y. S. Kim, C. -H. Hong, and H. J. Lee, M. S. Jeong, J. O. White, E. -K. Suh, "Yellow luminescence and persistent photoconductivity of undoped n-type GaN", J. Appl. Phys. 89 (10), 5454, 2001.

4. C. V. Reddy, K. Balakrishnan, H. Okumura, and S. Yoshida, "The origin of persistent photoconductivity and its relationship with yellow luminescence in molecular beam epitaxy grown undoped GaN", Appl. Phys. Lett. 73 (2), 244, 1998.

5. U. Kaufmann, M. Kunzer, H. Obloh, M. Maier, Ch. Manz, A. Ramakrishnan, and B. Santic, "Origin of defect-related photoluminescence bands in doped and nominally undoped GaN", Phys. Rev. B 59 (8), 5561 (1999).

6. Z. C. Huang, D. B. Mott and P. K. Shu, R. Zhang, and J. C. Chen, D. K. Wickenden, "Optical quenching of photoconductivity in GaN photoconductors", J. Appl. Phys. 82(5), 2707, 1997.

7. T. Y. Lin, H. C. Yang, and Y. F. Chen, "Optical quenching of the photoconductivity in $n$-type GaN", J. Appl. Phys. 87, 3404, 2000.

8. C, H. Qiu and J. I. Pankove, "Deep levels and persistent photoconductivity in GaN thin films", Appl. Phys. Lett. 70 (15), 1997.

9. H. M. Chen, Y. F. Chen, M. C. Lee, and M. S. Feng, "Persistent photoconductivity in $n$-type GaN", J. Appl. Phys. 82 (2), 899, 1997.

10. M.T. Hirsch, J. A. Wolk, W. Walukiewicz, and E. E. Haller, "Persistent photoconductivity in $n$-type GaN", Appl. Phys. Lett. 71 (8), 1098, 1997.

11. J. Z. Li, J. Y. Lin, H. X. Jiang, A. Salvador, A. Botchkarev, and H. Morkoc, "Nature of Mg impurities in GaN", Appl. Phys. Lett. 69 (10), 1474, 1996.

12. J. Z. Li, J. Y. Lin, H. X. Jiang, M. A. Khan, and Q. Chen, "Two-dimensional electron gas in AlGaN/GaN heterostructure", J. Appl. Phys. 82, 1227, 1997.

13. J. Z. Li, J. Y. Lin, H. X. Jiang, M. A. Khan, and Q. Chen, "Persistent photoconductivity in a two-dimensional electron gas system formed by an AlGaN/GaN heterostructure", J. Vac. Sci. Technol. B 15, 1117, 1997.

14. C. Johnson, J. Y. Lin, H. X. Jiang, M. A. Khan, and C. J. Sun, "Metastability and persistent photoconductivity in Mgdoped p-type GaN", Appl. Phys. Lett. 68, 1808, 1996.

15. G. Badei, W. S. Rabinovich, A. E. Wickenden, D. D. Koleske, S. C. Binari, and J. A. Freitas, J. Appl. Phys. 71, 1092, 1997.

16. W. Rieger, R. Dimitrov, D. Brunner, E. Rohrer, O. Ambacher, and M. Stutzmann, Phys. Rev. B 54, 17 596, 1996.

17. H. M. Chen, Y. F. Chen, M. C. Lee, and M. S. Feng, Phys. Rev. B 56, 6942, 1997.

18. S. Dhar and S. Ghosh, "Optical metastability in undoped GaN grown on Ga-rich GaN buffer layers", Appl. Phys. Lett. 80, 4519, 2002.

19. X. Zhang, P. Kung, A. Saxler, D. Walker, T. Wang, and M. Razeghi, Acta Phys. Pol. A. 88, 601, 1995.

20. E. R. Glaser, T. A. Kennedy, K. Doverspike, L. B. Rowland, D. K. Gaskill, J. A. Freitas, Jr. M. Asif. Khan, D. T. Olson, J. N. Kuznia, and D. K. Wickenden, Phys. Rev. B 51, 13326, 1995.

21. T. Ogino and M. Aoki, Jpn. J. Appl. Phys. Part 1 19, 2395, 1980.

22. A. Dissanayake, M. Elahi, H. X. Jiang, and J. Y. Lin, Phys. Rev. B 45, 13996, 1992. 
23. J. Y. Lin, A. Dissanayake, G. Brown, and H. X. Jiang, Phys. Rev. B 42, 5855, 1990.

24. M. De Vittorio, B. Potì, M. T. Todaro, M. C. Frassanito, A. Pomarico, A. Passaseo, M. Lomascolo and R. Cingolani, "High temperature characterization of GaN based photodetectors", to be published on Sens. Actuators.

25. T. L. Tansley and R. J. Egan, "Point-defect energies in the nitrides of aluminium, gallium, and indium", Phys. Rev. B 45, $10942,1992$.

26. V. Bougrov, M. E. Levinshtein, S. L. Rumyantsev, A. Zubrilov, Properties of Advanced Semiconductor Materials GaN, AlN, InN, BN, SiC, SiGe . Eds. Levinshtein M. E., Rumyantsev S. L., Shur M. S., John Wiley \& Sons, Inc., New York, 1-30, 2001.

27. I. Gorczyca, A. Svane, N. E. Christensen, MRS Internet J. Nitride Semicond. Res. 2, 18, 1997.

28. J. Neugebauer and C. G. Van de Walle, "Gallium vacancies and the yellow luminescence in GaN", Appl. Phys. Lett. 69, 503, 1996.

29. S. M. Sze, Physics of Semiconductor Devices, 2nd ed. (Wiley, New York), 1981.

30. E. Munoz, E. Monroy, J. A. Garrido, I. Impura, F. J. Sanchez, M. A. Sanchez-Garcia and E. Calleja, "Photocurrent gain mechanisms GaN ultraviolet detectors", Appl. Phys. Lett. 71 (7), 870, 1997. 